\title{
Plasmonic Titania Photocatalysts Active under UV and Visible-Light Irradiation: Influence of Gold Amount, Size, and Shape
}

\author{
Ewa Kowalska, ${ }^{1,2,3}$ Sven Rau, ${ }^{1}$ and Bunsho Ohtani ${ }^{3}$ \\ ${ }^{1}$ Institute of Inorganic Chemistry I, Ulm University, Albert-Einstein-Allee 11, $89081 \mathrm{Ulm}$, Germany \\ ${ }^{2}$ Institute of Inorganic Chemistry, Friedrich-Alexander University of Erlangen-Nuremberg, Egerlandstraße 1, 91058 Erlangen, Germany \\ ${ }^{3}$ Catalysis Research Center, Hokkaido University, North 21, West 10, Sapporo 001-0021, Japan
}

Correspondence should be addressed to Ewa Kowalska, ewa.kowalska@chemie.uni-erlangen.de

Received 29 February 2012; Accepted 28 March 2012

Academic Editor: Reda M. Mohamed

Copyright ( $\odot 2012$ Ewa Kowalska et al. This is an open access article distributed under the Creative Commons Attribution License, which permits unrestricted use, distribution, and reproduction in any medium, provided the original work is properly cited.

\begin{abstract}
Plasmonic titania photocatalysts were prepared by titania modification with gold by photodeposition. It was found that for smaller amount of deposited gold $(\leq 0.1 \mathrm{wt} \%)$, anatase presence and large surface area were beneficial for efficient hydrogen evolution during methanol dehydrogenation. After testing twelve amounts of deposited gold on large rutile titania, the existence of three optima for $0.5,2$ and $>6 \mathrm{wt} \%$ of gold was found during acetic acid degradation. Under visible light irradiation, in the case of small gold NPs deposited on fine anatase titania, the dependence of photoactivity on gold amount was parabolic, and large gold amount ( $2 \mathrm{wt} \%$ ), observable as an intensively coloured powder, caused photoactivity decrease. While for large gold NPs deposited on large rutile titania, the dependence represented cascade increase, due to change of size and shape of deposited gold with its amount increase. It has been thought that spherical/hemispherical shape of gold NPs, in comparison with rod-like ones, is beneficial for higher level of photoactivity under visible light irradiation. For all tested systems and regardless of deposited amount of gold, each rutile $\mathrm{Au} / \mathrm{TiO}_{2}$ photocatalyst of large gold and titania NPs exhibited much higher photoactivity than anatase $\mathrm{Au} / \mathrm{TiO} \mathrm{O}_{2}$ of small gold and titania NPs.
\end{abstract}

\section{Introduction}

Titanium(IV) oxide (titania) is one of the most commonly studied semiconductors due to its high photocatalytic activity, redox properties, thermal and chemical stability, and nontoxicity [1-3]. The limitation in its application, resulting from low quantum yield (fast recombination of charge carriers: $\mathrm{e}^{-} / \mathrm{h}^{+}$) and necessity to use UV irradiation, may be overcome since modified titania powders often possess higher level of activity and ability of working under visiblelight irradiation. A huge variety of organic [4-7] and inorganic compounds [8-10] have been examined as dopants or surface modifiers. Among them, noble-metals particles have attracted attention, since they may enhance the transfer of photogenerated electrons prolonging charge carriers lifetime [11-16]. Some of them, exhibiting plasmonic properties, such as gold and silver, may also activate wide band gap semiconductors, titania or ceria, towards visible light [1720].

Since Haruta's pioneering studies on catalytic (dark) properties of gold nanoparticles (NPs), a lot of studies have been carried out to explain nature of catalytic reaction and to find optimal conditions for efficient oxidation of organics $[21,22]$. Regarding catalytic and plasmonic properties of gold NPs the novel area of research on photocatalytic gold properties has been started [23-31].

In contrast with catalytic active gold NPs, where gold NPs must be nanosized [32, 33], larger gold NPs with high polydispersity in the size and shape are beneficial for photocatalytic activity under visible-light irradiation [18, $25,34]$. It is thought that wide size/shape distribution of gold nanoparticles and thus ability of absorption of light in a broad wavelengths range are responsible for the high level of photoactivity. The photocatalysts with the 
TABLE 1: Properties of bare and gold modified $\mathrm{TiO}_{2}$ photocatalysts.

\begin{tabular}{|c|c|c|c|c|c|c|c|c|}
\hline \multirow[t]{3}{*}{ Name } & \multicolumn{3}{|c|}{ Bare titania } & \multicolumn{5}{|c|}{ Titania after deposition of $2 \mathrm{wt} \%$ of gold ${ }^{* *}$} \\
\hline & \multirow[t]{2}{*}{$\mathrm{BET}^{*} / \mathrm{m}^{2} \mathrm{~g}^{-1}$} & \multirow[t]{2}{*}{ PPS* /nm } & \multirow[t]{2}{*}{ Form $^{\mathrm{a}}$} & \multirow[t]{2}{*}{ Color ${ }^{\mathrm{b}}$} & \multicolumn{2}{|c|}{ LSPR } & $\mathrm{PPS}^{\mathrm{d}} / \mathrm{nm}$ & Vis activity $/ \mu \mathrm{mol}$ \\
\hline & & & & & \multicolumn{3}{|c|}{$\lambda_{\max }{ }^{\mathrm{c}} \mathrm{nm}$} & \\
\hline ST-01 & 198 & 8 & A & d. pink & narrow & 538 & 9 & 0.07 \\
\hline TIO-10 & 100 & 15 & $\mathrm{~A}$ & d. violet & narrow & 558 & 12 & 0.08 \\
\hline Aldrich_A & 8 & 217 & $\mathrm{~A} / \mathrm{r}$ & 1. violet & broad & 567 & 34 & 0.28 \\
\hline ST-G1 & 5.7 & 250 & $\mathrm{R}$ & violet & broad & 580 & 43 & 0.29 \\
\hline Aldrich_R & 4 & 517 & $\mathrm{R} / \mathrm{a}$ & 1. grey & broad & 593 & 60 & 0.43 \\
\hline TIO-5 & 3 & 570 & $\mathrm{R} / \mathrm{a}$ & 1. grey & broad & 605 & 44 & 0.57 \\
\hline
\end{tabular}

Symbols ${ }^{*}$ and ${ }^{* *}$ correspond to data in [37] and [25], respectively. ${ }^{\mathrm{a}} \mathrm{A}$ : anatase, R: rutle, A/r: predominantly anatase, R/a: predominantly rutile, ${ }^{\mathrm{b}} \mathrm{d} .:$ dark, 1. : light, ${ }^{\mathrm{c}} \lambda_{\max }$ : LSPR wavelength at maximum absorption, ${ }^{\mathrm{d}} \mathrm{PPS}$ : primary particle size, ${ }^{\mathrm{e}}$ activity measured with mercury lamp and a Y48 cut-off filter ( $>470$ nm).

highest photoactivity were obtained by photodeposition of gold on titania with large primary particle sizes, that is, predominantly rutile crystalline form of $\mathrm{TiO}_{2}$. The mean size of generated gold NPs was directly proportional to the size of primary particle size of host titania. As a result the most active photocatalysts are characterized by small surface area. The activity loss has been also observed for these photocatalysts due to aggregation of large gold NPs [35]. In contradistinction with photocatalysts of lower level of photoactivity but high photostability, in which small gold NPs deposited on small titania NPs (anatase crystalline form of $\mathrm{TiO}_{2}$ ) were also partly covered by other titania NPs hindering gold aggregation.

In the present paper we will discuss the possibility of improvement of photoactivity of these photostable goldmodified anatase photocatalysts of small gold and titania NPs, influence of irradiation source on observable photoactivity, and optimal amount of deposited gold on photoactivity under UV and vis irradiation for anatase and rutile $\mathrm{Au} / \mathrm{TiO}_{2}$.

\section{Experimental}

2.1. Materials. Six commercial titania photocatalysts, ST01 (Ishihara, Japan), TIO-10, TIO-5 (Catalysis Society of Japan, Japan), ST-G1 (Showa Titanium, Japan), Aldrich_R and Aldrich_A (Aldrich), described in Table 1, were used as titania source. Hydrogen tetrachloroaurate(III) tetrahydrate $\left(\mathrm{HAuCl}_{4} \cdot 4 \mathrm{H}_{2} \mathrm{O}\right)$ (Nacalai Tesque and Wako Pure Chemical Industry) was used as received for metal loadings. Methanol, acetic acid, 2-propanol, acetone, a gold standard solution, hydrochloric acid $(\mathrm{HCl})$, and sodium hydroxide $(\mathrm{NaOH})$ (Wako Pure Chemical Industry) were used without further purification.

Gold $(2 \mathrm{wt} \%)$ was photodeposited on the surface of titania simultaneously in eight Pyrex tubes (58.6 mL/each). In each tube, $572 \mathrm{mg}$ of titania powder was suspended in $28.6 \mathrm{~mL} 50 \mathrm{vol} \%$ aqueous methanol with $\mathrm{HAuCl}_{4} \cdot 4 \mathrm{H}_{2} \mathrm{O}$ solution. The tube was purged of air with argon for at least $15 \mathrm{~min}$ and then sealed with a rubber septum. The absence of oxygen in the tubes was checked chromatographically before starting irradiation. The suspensions were simultaneously photoirradiated with a $400 \mathrm{~W}$ high-pressure mercury lamp under magnetic stirring $(500 \mathrm{rpm})$ in the irradiation system shown previously [36]. The temperature of the suspensions during photoirradiation was maintained at $298 \pm 5 \mathrm{~K}$ using a thermostatically controlled water bath. During the irradiation, the amount of generated hydrogen was measured every $15 \mathrm{~min}$ by gas chromatography (Shimadzu GC8A-IT equipped with a thermal conductivity detector; TCD). Thusobtained $\mathrm{Au} / \mathrm{TiO}_{2}$ photocatalyst was centrifuged, washed with methanol and at least three times with Mili-Q water, dried overnight at $393 \mathrm{~K}$, and ground in an agate mortar. For determination of influence of gold amount on photoactivity, photodeposition experiments of $0.05,0.1,0.5,0.75,1,1.5,2$, $2.25,3,4,6$, and $10 \mathrm{wt} \%$ of gold were also carried out.

2.2. Characterization of Photocatalysts. The morphology of gold photodeposited onto titania was observed by scanning transmission electron microscopy (STEM, Hitachi HD2000 ultrathin film evaluation system). $\mathrm{Au} / \mathrm{TiO}_{2}$ powders were dispersed in ethanol in an ultrasound bath for a few minutes and some drops of suspension were deposited on an amorphous carbon-covered reinforced copper grid (Ohken, types A and B). The samples were dried under vacuum overnight. The images were acquired at a wide range of magnification (70000-1800000) in normal, highresolution and ultrahigh-resolution modes with 2 or $3 \mathrm{~mm}$ working distances, $200 \mathrm{kV}$ accelerating voltage, and $30 \mu \mathrm{A}$ emission current. Secondary electron (SE), Z-contrast (ZC), and bright-field (BF) images were recorded.

To characterize the photoabsorption properties of modified photocatalysts, diffuse reflectance (DR) spectra were recorded and data were converted to obtain absorption spectra. The measurements were carried out on a Hamamatsu Photonics C7473-6 photonic multichannel analyzer using barium sulfate and bare titania powders as references.

X-ray diffraction (XRD) patterns were recorded on a diffractometer (Rigaku, RINT Ultima+) equipped with a graphite monochromator using copper $\mathrm{K}_{\alpha}$ radiation $(40 \mathrm{kV}$ tube voltage and $20-\mathrm{mA}$ tube current). Measurements were carried out with two scan speeds (2 and $\left.0.2^{\circ} \mathrm{min}^{-1}\right)$ and three scan ranges $\left(10-90^{\circ}, 35-40^{\circ}\right.$, and $\left.42-47^{\circ}\right)$, the slower one for narrower ranges being used for particle size 
determination. To determine primary particle sizes of gold deposits, XRD data were calculated using Scherrer's equation with appropriate corrections [37].

The amount of deposited gold was determined by flame atomic absorption spectroscopy (FAAS, Shimadzu AA$6200)$. The deposited gold $\left(25 \mathrm{mg}\right.$ as $\left.\mathrm{Au} / \mathrm{TiO}_{2}\right)$ was dissolved by aqua regia $(2 \mathrm{~mL})$ under magnetic stirring $(250 \mathrm{rpm})$ for $>1 \mathrm{~h}$, decanted after centrifugation, and poured into a $50 \mathrm{~mL}$ volumetric flask. The remaining white titania was rinsed twice with $1 \mathrm{~mol} \mathrm{~L}^{-1} \mathrm{HCl}(5 \mathrm{~mL})$ under magnetic stirring (10 min, $250 \mathrm{rpm}$ ) and centrifuged. The resultant supernatant was added to the aqua regia solution of gold followed by the addition of $1 \mathrm{~mol} \mathrm{~L}^{-1} \mathrm{HCl}$. Gold calibration solutions were prepared from a gold standard solution by diluting with $1 \mathrm{~mol} \mathrm{~L}^{-1} \mathrm{HCl}$.

\subsection{Photocatalytic Activity Tests}

2.3.1. Photoirradation under UV and/or Vis with a Mercury Lamp. A metal-loaded photocatalyst $(50 \mathrm{mg}$ ) was suspended in an aqueous solution of acetic acid or 2-propanol $(5 \mathrm{vol} \%, 5 \mathrm{~mL})$ and photoirradiated under magnetic stirring $(1000 \mathrm{rpm})$ in the setup used for gold photodeposition described above with the exception of the test-tube holder, which was changed to enable irradiation of 12 thinner tubes at the same time. For the test of visible-light-induced activity, the test-tube holder was changed to four test-tube holders (each holder containing two sample tubes) with one or two cut-off filters (L42, Y43, Y44, and Y48, Asahi Techno Glass) mounted in the irradiation window. During the irradiation of acetic acid solutions, a portion $(0.2 \mathrm{~mL})$ of the gas phase of the reaction mixture was withdrawn with a syringe and subjected to gas chromatographic analysis of carbon dioxide $\left(\mathrm{CO}_{2}\right)$ (Shimadzu GC-14B equipped with a flame ionization detector (FID) and a methanizer (Shimadzu MTN-1)).

2.3.2. Photoirradion under Vis with a Xenon Lamp. The activity of samples under vis irradiation was also examined using a xenon lamp set-up. A sample holder, the same as that used in a mercury lamp set-up, with two tubes was kept in a water bath thermostated at $298 \pm 5 \mathrm{~K}$. The contents were magnetically stirred and irradiated by a xenon lamp installed outside the water bath. During the irradiation of 2-propanol solutions, generated acetone was gas-chromatographically analyzed by Shimadzu GC-14B equipped with an FID. Before injection of a portion of liquid phase to GC, the photocatalyst powder was separated from the suspension using a filter (Whatman Mini-UniPrep, PVDF).

The intensity of irradiation was measured by Ushio spectro-radiometer USR-30.

\section{Results and Discussion}

Recently, we have shown that after deposition of $2 \mathrm{wt} \%$ of gold on fifteen commercial titania samples the highest level of photoactivity under visible light irradiation exhibited samples with low BET surface area (i.e., 3-4 $\mathrm{m}^{2} \mathrm{~g}^{-1}$ ) and bright grayish color (see Table 1). Though the reason for their high photoactivity, that is, broad localized surface plasmon resonance (LSPR), is obvious, the low level of photoactivity of intensively colored, violet photocatalyst with large surface area $\left(\right.$ BET $\left.>200 \mathrm{~m}^{2} \mathrm{~g}^{-1}\right)$ is still questionable. To check whether these photocatalysts are practically inactive under visible-light irradiation or some experimental procedure/conditions were the cause of the unexpected findings, a few process modifications have been carried out and some of them had been already mentioned [25]. Presently we are discussing in detail how the photoactivity of these photocatalyst can be demonstrated and improved.

3.1. Broadening of Radiation Ranges. To test plasmonic activity of $\mathrm{Au} / \mathrm{TiO}_{2}$ photocatalysts, the activity of bare titania should be eliminated allowing only visible radiation to enter reaction suspension. To achieve this requirement an Y48 cut-off filter eliminating all radiation shorter than $470 \mathrm{~nm}$ was usually applied (light emission spectra of mercury and xenon lamps with and without cut-off filters were shown previously [25]). However application of this filter (Y48) makes it impossible to observe photoactivity caused by small gold NPs (less than $10 \mathrm{~nm}$ ); for example, nanosized gold $(<2 \mathrm{~nm})$ absorbs light at $410 \mathrm{~nm}$ [38]. In this regard, observable very low photoactivity of $\mathrm{Au} / \mathrm{TiO}_{2}$ composed of small gold NPs could be caused by irradiation with insufficient light (too long wavelengths) to show plasmonic properties of these small gold NPs. To determine whether anatase $\mathrm{Au} / \mathrm{TiO}_{2}$ : (ST-01, TIO-10) exhibit a higher level of activity at shorter wavelengths, the photodegradation of 2propanol to acetone was repeated for a wider wavelength range using other cut-off filters: Y43 (>420 nm) and L42 $(>390 \mathrm{~nm})$. Obtained results are shown in Figure 1. Indeed broadening of radiation range increased the photoactivity of $\mathrm{Au} / \mathrm{TiO}_{2}$ (ST-01). However, the obtained photoactivity was still much lower than the photoactivity of best rutile photocatalyst-Au/ $/ \mathrm{TiO}_{2}$ (Aldrich_R), even for much narrower wavelength ranges $(\lambda>470 \mathrm{~nm})$.

Obtained results were also checked for more constant irradiation source, that is, xenon lamp, for three irradiation ranges: $\lambda>430 \mathrm{~nm}$ (two Y44 cut-off filters), $\lambda>450 \mathrm{~nm}$ (two Y46 cut-off filters), and $\lambda>470 \mathrm{~nm}$ (two Y48 cutoff filters), for four bare and gold modified photocatalysts, that is, $\mathrm{TiO}_{2}$ : ST-01, TIO-10, ST-G1, and Aldrich_R (Figure 2 ). The obtained acetone-generation rates increased with broadening of irradiation wavelengths for all tested photocatalysts. However, the highest increase was observed for the most active photocatalyst- $\mathrm{Au} / \mathrm{TiO}_{2}$ (Aldrich $\_$R) - and thus photoactivity ratio of $\mathrm{Au} / \mathrm{TiO}_{2}$ (Aldrich_R) to $\mathrm{Au} / \mathrm{TiO}_{2}$ (ST01 ) also increased amounting to 3.1, 3.8, and 4.1 for irradiation wavelengths $>470,450$, and $430 \mathrm{~nm}$, respectively, confirming the highest photoactivity of $\mathrm{Au} / \mathrm{TiO}_{2}$ (Aldrich_R) with large gold and titania NPs. These results also show that participation of small gold NPs $(<20 \mathrm{~nm})$, which are in the minority (10 vol\% [25]), in the observable photoactivity, is significant (twice increase of photoactivity). It may indicate that spherical/hemispherical shape of small gold NPs could be responsible for higher level of photoactivity than gold NPs of rod-like shape (rod-like shape observed by STEM 


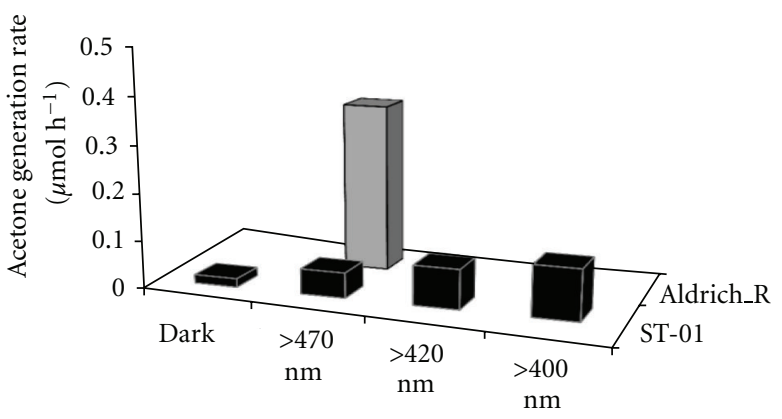

FIGURE 1: Comparison of rate of photocatalytic acetone generation from suspensions containing 2-propanol and $\mathrm{Au} / \mathrm{TiO}_{2}$ (ST-01 and Aldrich_R) under vis irradiation with mercury lamp and cut-off filters: Y48 $(\lambda>470 \mathrm{~nm}), \mathrm{Y} 43(\lambda>420 \mathrm{~nm}), \mathrm{L} 42(\lambda>400 \mathrm{~nm})$.

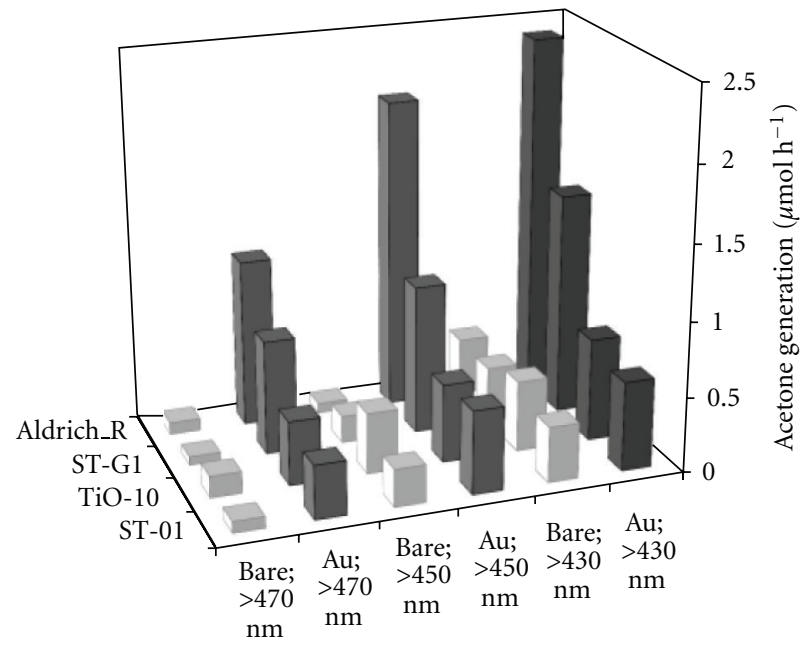

FIGURE 2: Comparison of rate of photocatalytic acetone evolution from suspensions containing 2-propanol and bare or gold modified titania photocatalysts (ST-01, TIO-10, ST-G1, and Aldrich_R) under vis irradiation with xenon lamp and cut-off filters: Y48 $(\lambda>$ $470 \mathrm{~nm})$, Y46 $(\lambda>450 \mathrm{~nm})$, Y44 $(\lambda>430 \mathrm{~nm})$.

and DRS as two kinds of plasmon resonance: transverse and longitudinal LSPR at 537 and $580 \mathrm{~nm}$, resp. [25]). This observation is contradictory to recent results by Yuzawa et al. where rod-like NPs were probably responsible for higher level of photoactivity than spherical ones during hydrogen generation from aqueous ethanol suspension [39]. However, party elimination of the photoactivity of small, spherical gold NPs could not be excluded, since the photoactivity level was checked during irradiation with long wavelengths $(>510 \mathrm{~nm})$. Moreover, the most active photocatalysts were obtained by photodeposition method, for which controlling the particle size/shape is difficult, and thus the presence of undesirable NPs cannot be excluded; that is, the authors observed both spherical and rod-like gold NPs in the most active samples.

It is worth mentioning that broadening of irradiation ranges caused also increase of photoactivity of bare titania.
For the photocatalysts with the smallest photoactivities (ST01 and TIO-10), the photoactivities of bare and modified photocatalysts were almost the same for wavelengths longer than $430 \mathrm{~nm}$. This was caused partly by UV excitation even when two cut-off filters were used as shown in Figure 3. In this regard, the use of narrower irradiation ranges is recommended for testing plasmonic titania photocatalysts, that is, wavelengths longer than $470 \mathrm{~nm}$. However, it must be remembered that the photoactivity caused by small gold NPs would be undetectable under irradiation with such long wavelengths.

3.2. Change of Irradiation Source. In the case of plasmonic photocatalysts the selection of irradiation wavelengths is crucial. For testing visible activity, elimination of influence of NPs support (often active photocatalyst under UV irradiation, such as titania (shown above)) must be performed. It can be achieved by use of [36]:

(i) UV/vis emitting lamps, for example, mercury or xenon lamps, equipped with IR and UV cut-off filters, preferentially with transmission of only visible light (see Section 3.1.),

(ii) visible-light-emitting lamps, for example, tungsten and halogen lamps, equipped with UV cut-off filters ( since they may emit also small part of UV),

(iii) light-emitting diodes (LEDs).

The UV/vis or vis lamps (two former groups) are recommended for comparison of photoactivity of different plasmonic materials since the photoactivity level depends on wavelengths; that is, NPs size and shape influence the position of LSPR, while application of LEDs seems more reasonable for action spectrum analysis.

In our experiments the same set-up as for gold photodeposition was preliminary used; that is, mercury lamp, with a sample holder equipped with UV cut-off filter immersed in a water bath (an IR filter). It is thought that unevenness of emission spectra of a mercury lamp, which emits four main intense lines in visible range at 405, 436, 547, and $579 \mathrm{~nm}$, results in mismatch with gold absorption and thus being the reason for the lower level of photoactivity of small anatase powders. For example, 18 times stronger irradiation intensity at $580 \mathrm{~nm}$ than at $558 \mathrm{~nm}(218$ and $12 \mu \mathrm{W} \mathrm{cm}$, resp.) could be a reason for 3.7 lower photoactivity of anatase $\mathrm{Au} / \mathrm{TiO}_{2}(\mathrm{TIO}-10)$ than rutile $\mathrm{Au} / \mathrm{TiO}_{2}$ (ST-G1) for which maxima LSPR values are observed at 558 and $580 \mathrm{~nm}$, respectively.

In this regard, experiments for five $\mathrm{Au} / \mathrm{TiO}_{2}$ photocatalysts (ST-01, TIO-10, Aldrich_A, ST-G1, and Aldrich_R) under visible-light irradiation with a radiation source of more evener emission, that is, a xenon lamp (e.g., 290 and $251 \mu \mathrm{W} \mathrm{cm} \mathrm{cm}^{2}$ at 580 and $558 \mathrm{~nm}$, resp., see Figure 3 ), were carried out. Irradiation with more intense light of power led to a marked increase in the reaction rate for all tested powders, and the fastest 2-propanol oxidation was still obtained for large rutile powder, that is, $2.57 \mu \mathrm{mol} \mathrm{h}^{-1}$ by $\mathrm{Au} / \mathrm{TiO}_{2}$ (Aldrich_R), as shown in Figure 4 . The activity levels of small anatase powders were still very low, that is, 0.36 


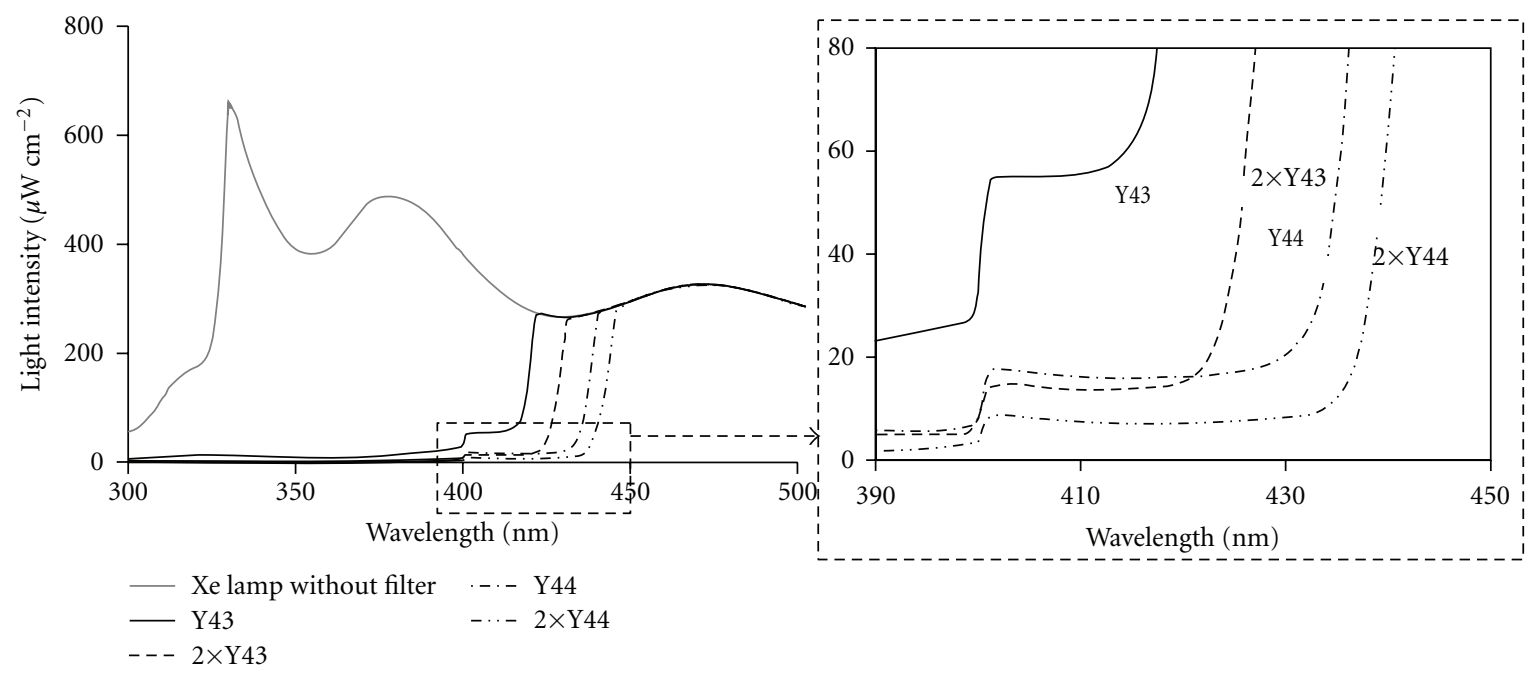

FIGURE 3: Light emission spectra of xenon lamp with one or two cut-off filters: Y43, Y44.

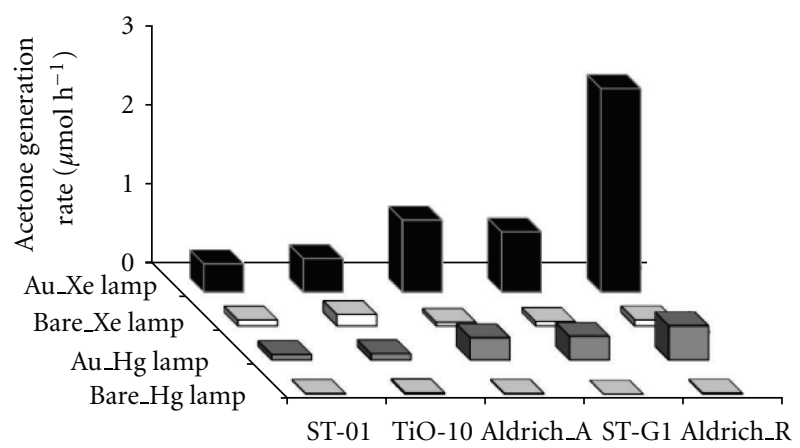

FIGURE 4: Comparison of rate of photocatalytic acetone evolution from suspensions containing 2-propanol and bare or gold modified titania photocatalysts (ST-01, TIO-10, Aldrich_A, ST-G1 and Aldrich_R) under vis irradiation with a mercury or a xenon lamp and cut-off filter: Y48 (>470 nm).

and $0.42 \mu \mathrm{mol} \mathrm{h}^{-1}$ for $\mathrm{Au} / \mathrm{TiO}_{2}$ (ST-01), and $\mathrm{Au} / \mathrm{TiO}_{2}$ (TIO10), respectively. The largest enhancement of activity by changing the light source from mercury to xenon lamp was observed for large rutile and small anatase powders, that is, 6.4, 5.2, and 5.3 times for Aldrich_R, ST-01, and TIO10 , respectively. On the other hand, the enhancement ratio of ST-G1 and Aldrich_A, for which LSPR was observed at almost the same wavelength as that of main mercury lamp emission, was only 3 times. Therefore, to obtain actual data of activity for plasmonic photocatalysts possessing LSPR at different wavelengths, the use of an irradiation source with even intensity in the whole irradiation range, for example, xenon lamps, is recommended.

3.3. Decrease of Gold Amount. Though photoactivity increase of anatase $\mathrm{Au} / \mathrm{TiO}_{2}$ under irradiation with xenon lamp was observed, the activity was still much lower than photoactivity of best rutile photocatalysts. Thus, an excess amount of deposited gold on these small photocatalysts, resulting in very dark color, was considered to be a possible reason of their low level of activity, as a result of reduced penetration depth and increased scattering of the incident light beam [40-43].

3.3.1. Photocatalysts Preparation. To prepare photocatalysts with higher level of activity, much smaller amounts of gold $(0.05,0.1 \mathrm{wt} \%)$ were deposited on two small anatase and on one rutile titania photocatalysts: ST-01, TIO-10, and Aldrich_R, respectively. To find an optimal amount of gold, rutile photocatalysts with various amounts of gold, that is, $0.05,0.1,0.5,0.75,1,1.5,2,2.25,3,4,6$, and $10 \mathrm{wt} \%$, were also prepared.

During gold photodeposition in the presence of methanol as a sacrificial hole scavenger under UV photoirradiation, continuous liberation of hydrogen at a constant rate was observed after short induction period ( $<15 \mathrm{~min})$ for all of the samples. During the induction period gold cations were reduced to metallic gold NPs. Typical hydrogen evolution curve during gold photodeposition was shown in previous report [25]. Continuous hydrogen evolution also means, from our experience on metal photodeposition on titania, that all precursor gold ions had been reduced to metallic gold $[14,25,44]$. Deposition of whole gold precursor was also confirmed by FAAS and exemplary results are shown in Figure 5.

Thus, photodeposition method confirms to be more profitable than other methods, for example, impregnationreduction [25], deposition-precipitation [28, 45], deposition-calcination [28, 45], microemulsion [34], in which only some part of gold precursor is deposited on the support. In addition, the process is very short, and 15 minutes is sufficient for deposition of whole gold precursor, independently on used amount over a wide concentration range $(0.05-10 \mathrm{wt} \%)$.

The fastest hydrogen evolution was observed on gold deposited on small anatase titania which is consistent with 


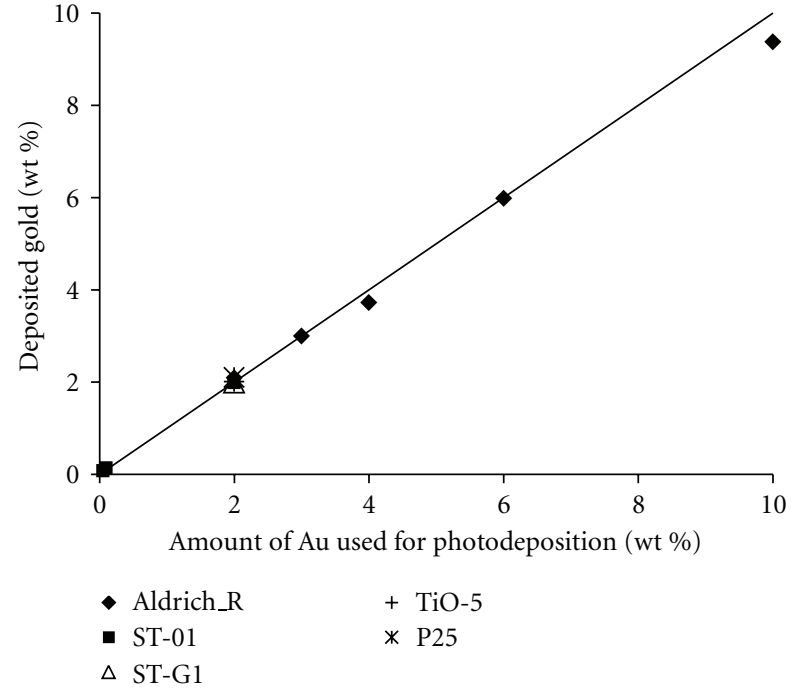

Figure 5: Correlation between gold amounts used for photodeposition and determined in $\mathrm{Au} / \mathrm{TiO}_{2}$ samples by FAAA.

our previous studies indicating that anatase crystallite form and large surface area promote alcohol dehydrogenation $[25,37]$. Previously we also found that increase of surface area accelerated hydrogen evolution only in the case of gold deposition on anatase, while in the case of rutile samples, the smaller the surface area, the better the photocatalytic activity. Thus, similar rates of hydrogen evolution on gold NPs deposited on small anatase and on large rutile titania were observed in the case of $2 \mathrm{wt} \%$ of gold loading. Figure 6 presents results of hydrogen evolution for wide range of gold amount, that is, $0.05-10 \mathrm{wt} \%$. It is clearly observable that even very small amount of deposited gold (0.05 wt $\%)$ is efficient for high rate of hydrogen evolution on small anatase photocatalysts. The dependence of reaction rate on BET surface area is shown in the inset of Figure 6. It has been found that, in the case of small amount of deposited gold, that is, 0.05 and $0.1 \mathrm{wt} \%$, the surface area is a key factor for efficient alcohol dehydrogenation, while in the case of larger amount of deposited gold, that is, $2 \mathrm{wt} \%$, surface area does not influence the efficiency significantly, that is, 50 times increase of BET causes only 1.12-fold increase of reaction rate.

3.3.2. Photocatalysts Characterization. Absorption spectra of anatase $\mathrm{Au} / \mathrm{TiO}_{2}$ (ST-01 and TIO-10) indicate that regardless of the amount of deposited gold (0.05, 0.1 and $2 \mathrm{wt} \%$ ) similar NPs (size and shape) are formed on the support, that is, the same position of LSPR, as shown in Figure 7(a). Similarly, in the case of rutile titania (Aldrich_R), rod-like gold NPs, observable by transverse and longitudinal LSPR at 537 and $580 \mathrm{~nm}$, respectively, are formed even at very low amount of deposited gold $(0.05 \mathrm{wt} \%)$, as shown in Figure 7(b). However, at very large amount of deposited gold ( $\geq 6 \mathrm{wt} \%$ ), disappearance of longitudinal LSPR was observed. It was expected that, with gold loading increase, larger gold NPs should be formed resulting in bathochromic shift of LSPR. Generation of larger gold NPs was confirmed by XRD analysis (Figure 8 ). Thus change of gold shape from rod-like to more spherical should be a reason of observable hypsochromic shift of LSPR.

The microscopic images of anatase and rutile $\mathrm{Au} / \mathrm{TiO}_{2}$ (ST-01 and Aldrich_R) for the smallest (0.05 wt\%) and largest $(10 \mathrm{wt} \%)$ amount of deposited gold are shown in Figures 9 and 10, respectively. In the case of anatase sample, regardless of the amount of deposited gold, gold NPs are very similar, that is, spherical of mainly $11-20 \mathrm{~nm}$ size, as was expected from DRS data. Comparison of gold distribution for two photocatalyst with 0.05 and $2 \mathrm{wt} \%$ gold loading is shown in Figure 9(b). In the case of large rutile photocatalysts, larger and more spherical gold NPs were formed, as was expected from DRS and XRD results. However, large, shapeless gold objects, unexpected from DRS data, were also formed, due to gold aggregation.

3.3.3. Photoactivity under UV/Vis Irradiation. Photoactivity of these samples under UV/vis irradiation during acetic acid oxidation was checked and data are shown in Figure 11. Modification of titania with even very small amount of gold, that is, $0.05 \mathrm{wt} \%$, increased photoactivity significantly ( $c a$. twice). This behaviour has been well known and reported as enhancement of separation of charge carriers $\left(\mathrm{e}^{-} / \mathrm{h}^{+}\right)$since noble metals serve as electron sink [12]. For all photocatalysts, increase of gold loading, from 0.05 to $2 \mathrm{wt} \%$, causes increase of photoactivity. Three maxima were observed for rutile $\mathrm{Au} / \mathrm{TiO}_{2}$ at $0.5,2$, and $\geq 6 \mathrm{wt} \%$. The reason for a few maxima is unclear. The dependence of UV photoactivity on amount of deposited gold had been already studied and an existence of optimal amount of loaded gold for example, 0.5 and $0.8 \mathrm{wt} \%$ for formic acid and dye (acid green 16) degradation, respectively, was reported [46, 47, 50]. It was suggested that excess loading of gold (i) covered active sites on titania surface which act as oxygen photoactivation centers [46] or (ii) led to formation of large gold NPs which act as recombination centres for photogenerated chargle carriers $\left(\mathrm{e}^{-} / \mathrm{h}^{+}\right)$[47]. The decrease [48] or increase [49] of photoactivity with gold loading was also reported. However, only a few gold amounts were usually tested, that is, five $(0.06,0.13,0.38,0.5,2.5 \mathrm{wt} \%)$ [50], four $(0.4,0.8,1.2$, $1.6 \mathrm{wt} \%)$ [46], and four $(0.1,1,4,8 \mathrm{wt} \%)$ [49]. The existence of three maxima shown in present report for twelve samples of deposited gold $(0.05,0.1,0.5,0.75,1,1.5,2,2.25,3,4$, $6,10 \mathrm{wt} \%$ ) seems novel, and a few opposing factors could be responsible for this behaviour, that is, increase of gold amount may cause (i) enhancement of separation of charge carriers, (ii) decrease of active surface area, (iii) reduction of light penetration depth, (iv) increase of visible photoactivity by plasmonic gold NPs, and (v) change of size and shape of gold NP. In this regard, more studies are necessary to confirm/exclude the influence of individual parameters.

3.3.4. Photoactivity under Visible-Light Irradiation $(>470 \mathrm{~nm})$. In the case of small anatase photocatalysts the decrease in the amount of deposited gold to 0.05 or $0.1 \mathrm{wt} \%$ resulted in brightly colored powders of higher 


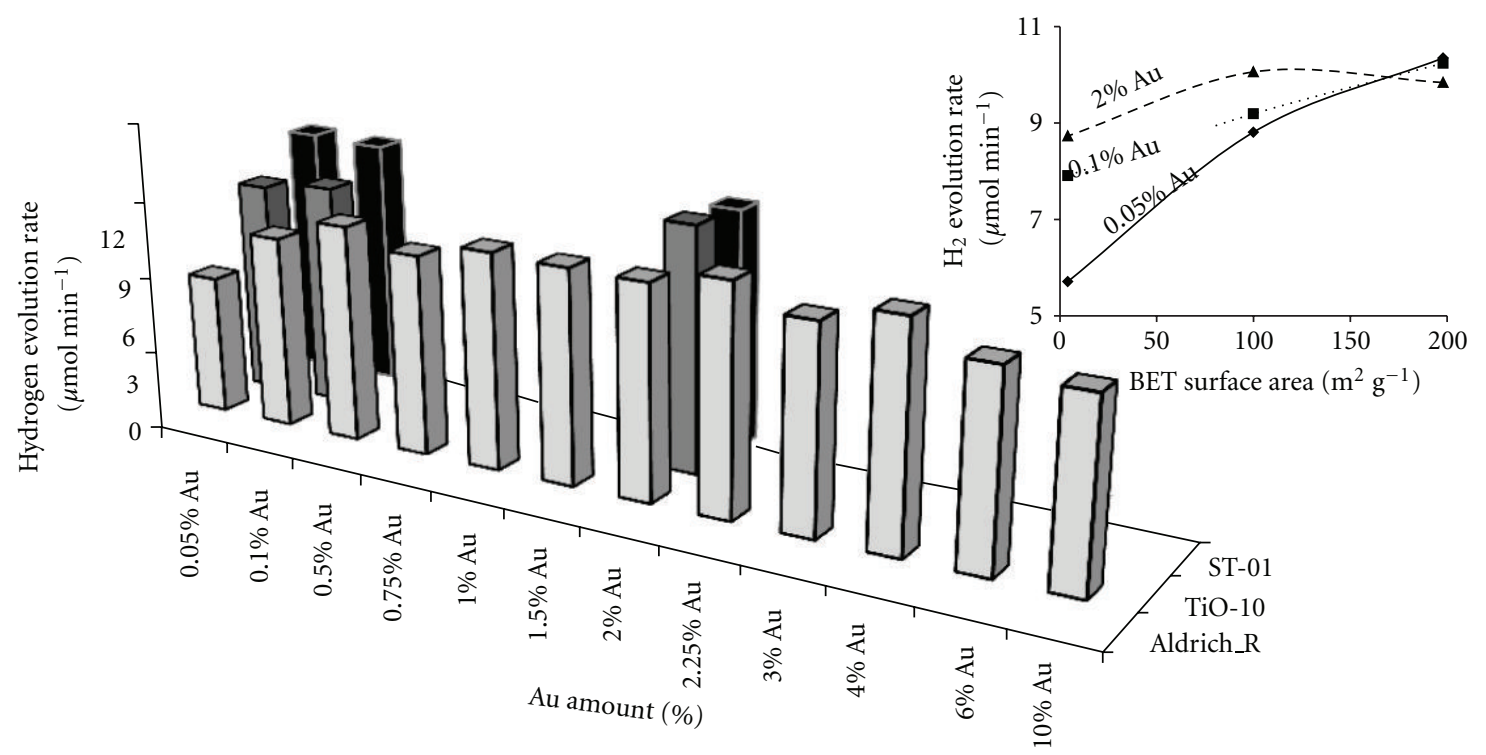

FIGURE 6: Influence of gold amount on methanol dehydrogenation during photodeposition on three titania photocatalysts: ST-01, TIO-10, Aldrich_R (under UV/vis with mercury lamp). Inset. Dependence of hydrogen evolution on BET surface area $\left(198,100\right.$, and $4 \mathrm{~m}^{2} \mathrm{~g}^{-1}$ for ST-01, TIO-10, and Aldrich_R, resp.) for three amounts of gold used for deposition: $0.05,0.1$, and $2 \mathrm{wt} \%$.

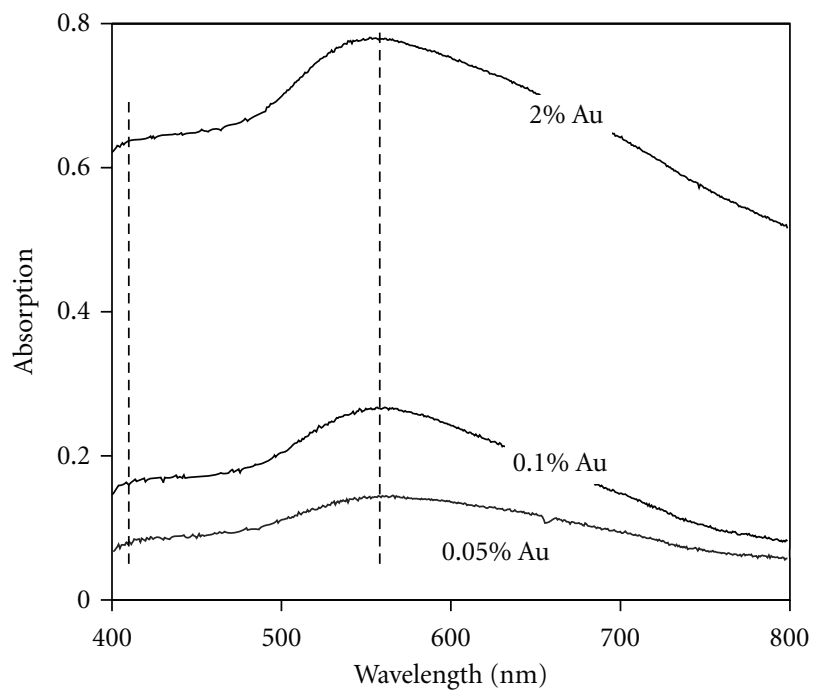

(a)

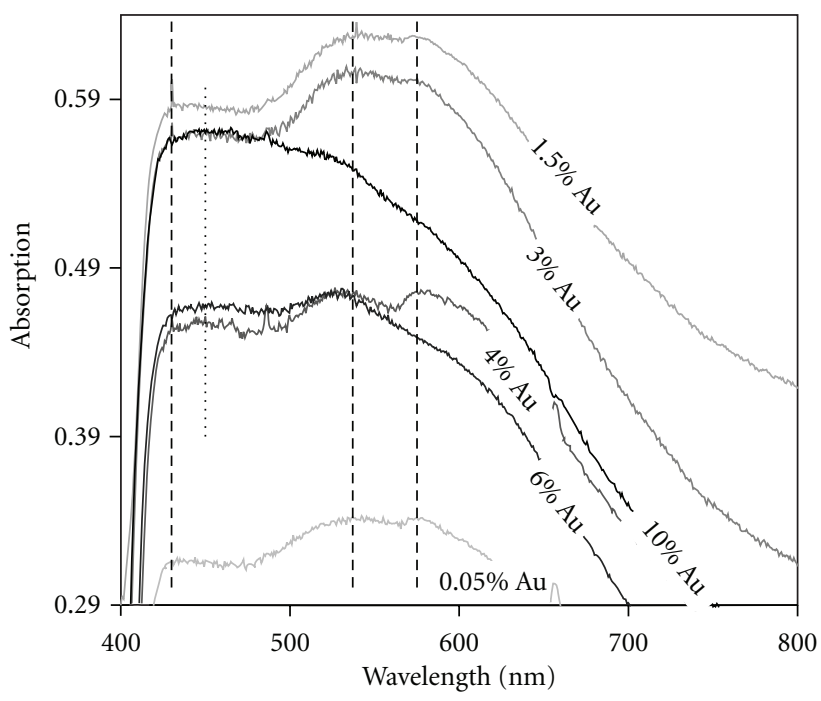

(b)

Figure 7: Exemplary DRS of Au/TiO 2 : TIO-10 (a) and Aldrich_R (b) for different amounts of deposited gold.

level of activity as shown in Figure 12 (a). The increase of photoactivity with decrease of amount of deposited gold is significantly observable for the ST-01 photocatalyst, that is, twice photoactivity increase with twenty-fold decrease of gold amount. The parabolic shape of dependence illustrates that an optimal gold amount must be much lower than usually used, that is, $2 \mathrm{wt} \%$, and its overflow results in significant activity decrease, possibly due to reduced penetration depth of light. Similar results, that is, photocatalytic yield decrease with the increase of $\mathrm{Au}$ loading and absorption intensity, were presented by Yuzawa et al. during ethanol dehydrogenation under visible-light irradiation [39]. It was proposed that inefficient use of photoexcitated electrons in Au NPs after increase of $\mathrm{Au}$ loading, resulting from the increase of Au NPs size, and the decrease of the number density of the Au NPs, was the reason of yield decrease.

Though the photoactivity of small anatase $\mathrm{Au} / \mathrm{TiO}_{2}$ increased with gold amount decrease, the photoactivity of large rutile $\mathrm{Au} / \mathrm{TiO}_{2}$ was still much higher, regardless of the amount of gold used for deposition. The detailed dependence of photoactivity on deposited amount of gold is shown in Figure 12 (right). It has been found that even very low amount of gold, that is, $0.05 \mathrm{wt} \%$, causes visible activity, 


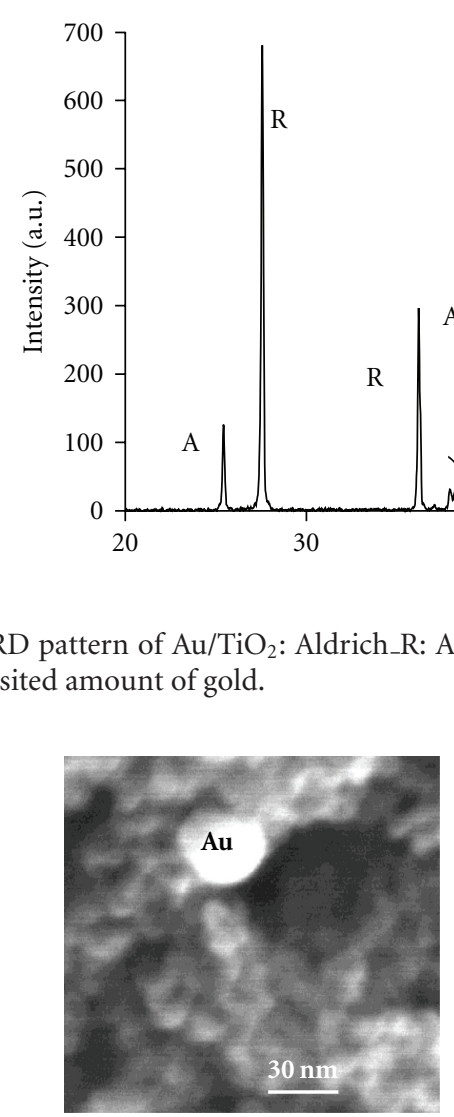

(a)

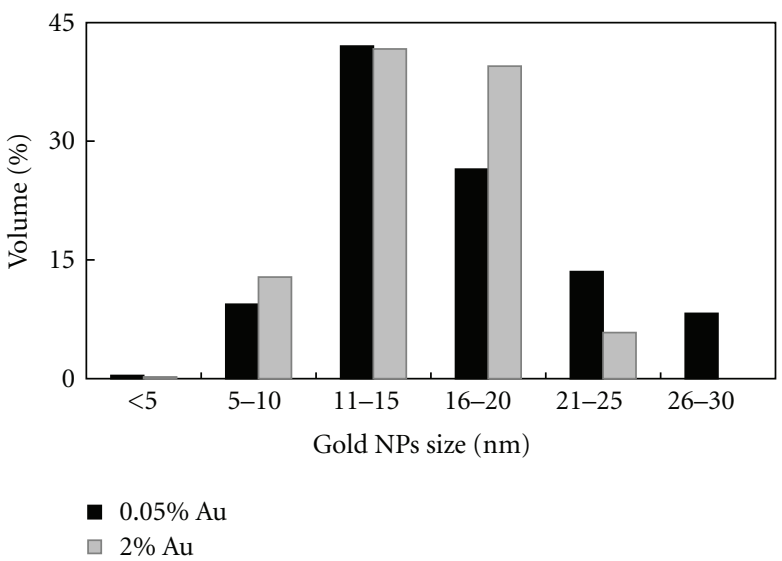

(b)

Figure 9: STEM image of $\mathrm{Au} / \mathrm{TiO}_{2}$ : ST-01 $(0.05 \mathrm{wt} \% \mathrm{Au})$ and comparison of distribution of gold NPs sizes for two amounts $(0.05$ and $2 \mathrm{wt} \%)$ of deposited gold on anatase ST-01 titania.

much higher than activity of anatase photocatalysts. Further increase of gold amount caused photoactivity increase by only $20 \%$ and then was constant, regardless of gold amount for $0.5-2.25 \mathrm{wt} \%$. Subsequent increase of gold amount from 2.25 to $4 \mathrm{wt} \%$ caused photoactivity increase by $27 \%$ then was constant till $6 \mathrm{wt} \% \mathrm{Au}$ and once more increased by $60 \%$ for the largest amount of deposited gold $(10 \mathrm{wt} \%)$. The obtained results of cascade shape were graphically divided into three groups: A, B, and C, characterized by photoactivity increase followed by constant rate (Figure 12 (b)). The first range A is very similar to well-known dependence of photocatalytic reaction rate on amount of photocatalyst, that is, increase of reaction rate due to increase of absorbed photon flux followed by constant rate due to optimal light absorption [41, 43]. Similarly in our case with increase of amount of deposited gold more gold NPs are formed and thus more photons could be absorbed till plateau region (from 0.5 to $2.25 \mathrm{wt} \% \mathrm{Au}$ ) indicating that optimal conditions is reached. Range B is different from classical dependence and illustrates that further increase of reaction rate is possible. This increase is caused by change of gold NPs shape and size as it was demonstrated by DRS (Figure 7(b)), XRD (in the inset of Figure 8), and STEM (Figure 10). The formation of larger gold NPs with different absorption/scattering properties resulted in higher level of photoactivity reaching again plateau for $4-6 \mathrm{wt} \%$ Au. Range $\mathrm{C}$ demonstrates further increase of photoactivity with increase of gold amount. Disappearance of rod-like gold NPs (lack of longitudinal LSPR) and formation of larger and more spherical/hemispherical NPs are probably responsible for the higher level of photoactivity. However, the influence of shapeless, large gold deposits (Figure 10) cannot be excluded.

\section{Conclusions}

Photodeposition of gold on titania surfaces allows to obtain active photocatalysts under both UV and vis irradiation. 


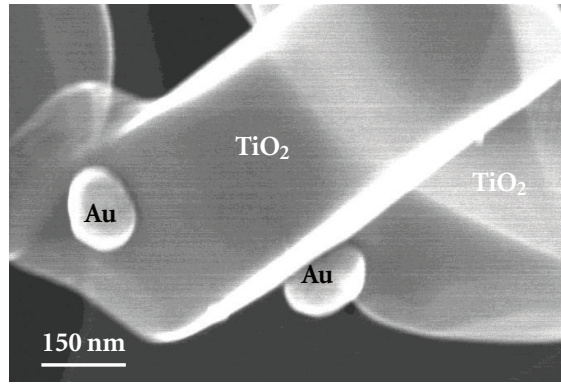

(a)

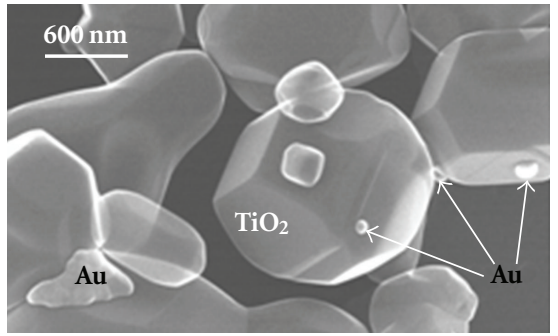

(c)

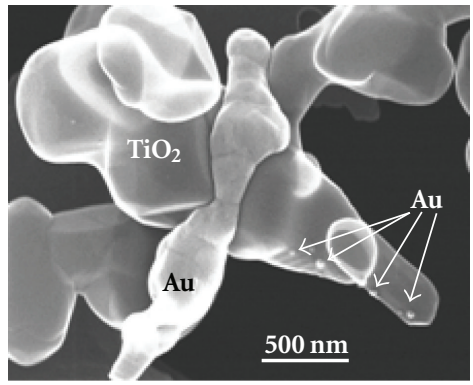

(b)

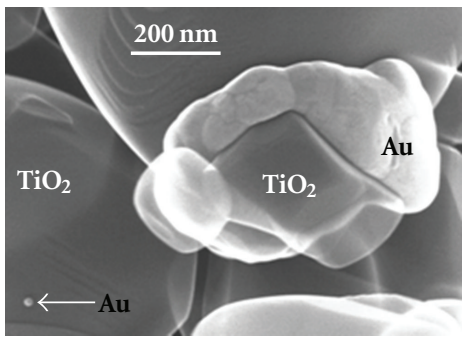

(d)

FIgure 10: STEM images of $\mathrm{Au} / \mathrm{TiO}_{2}$ : Aldrich_R (10 wt $\left.\% \mathrm{Au}\right)$.

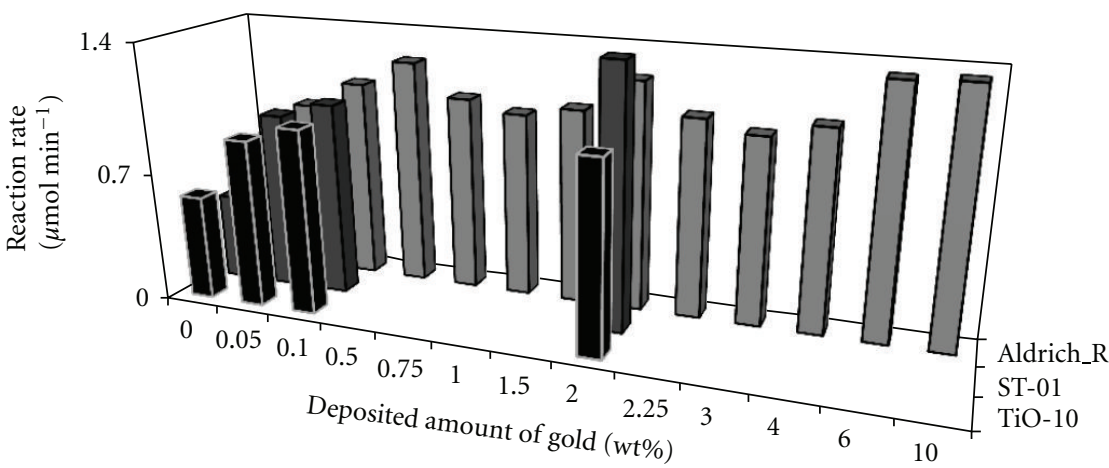

FIGURE 11: UV/vis photoactivity of gold modified titania photocatalysts: ST-01, TIO-10, and Aldrich_R during acetic acid oxidation.

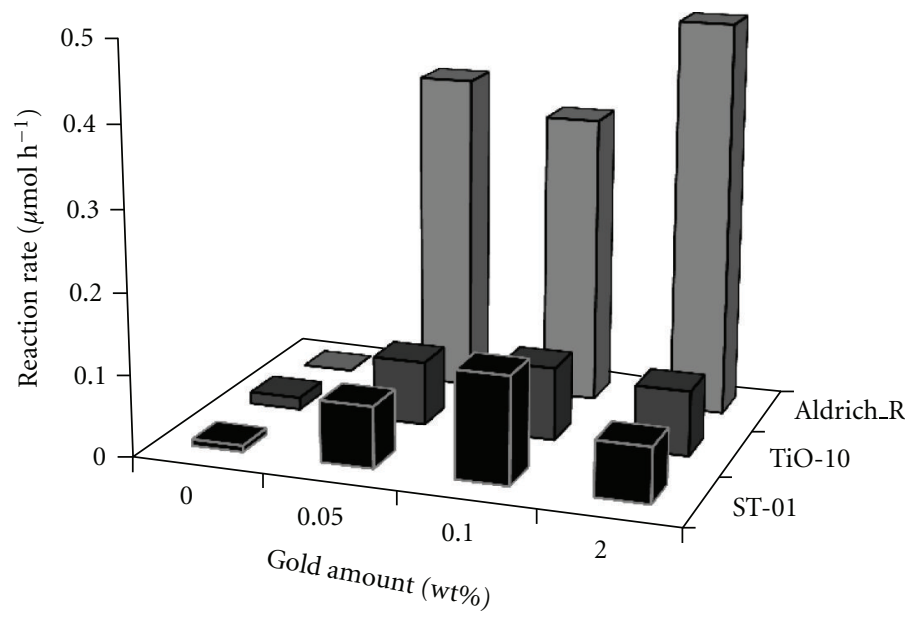

(a)

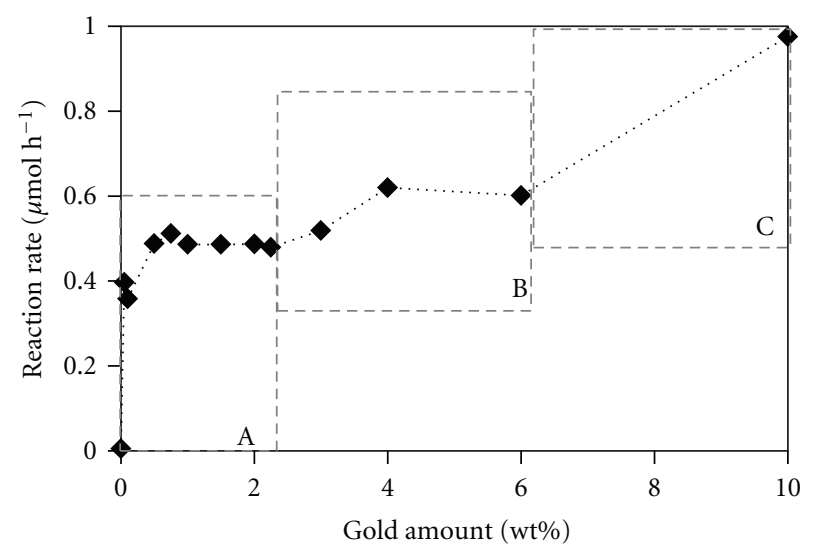

(b)

FIGURE 12: Dependence of deposited gold amount on acetone generation during visible light irradiation on $\mathrm{Au}_{\mathrm{TiO}}$ : ST-01, TIO-10, and Aldrich_R. (b) Detailed dependence for $\mathrm{Au} / \mathrm{TiO}_{2}$ : Aldrich_R. 
Anatase form and large surface area are preferable for methanol dehydrogenation during gold deposition. It has been found that this dependence is more evident for smaller amount of deposited gold $(0.05 \mathrm{wt} \%)$. The amount of deposited gold strongly influences the UV/vis photoactivity for acetic acid oxidation, and three photodegradation maxima are observed for $0.5,2$, and $>6 \mathrm{wt} \%$ of gold. Under visible-light irradiation it has been shown that participation of minor, small gold NPs in photoactivity of large rutile $\mathrm{Au} / \mathrm{TiO}_{2}$ (Aldrich_R) is disproportionately high, probably due to higher photoactivity of spherical/hemispherical gold NPs than rod-like ones.

For reliable comparison of visible photoactivity of plasmonic photocatalysts it is important to

(i) separate UV irradiation by sufficient cut-off filters eliminating the influence of support (e.g. $\mathrm{TiO}_{2}$ );

(ii) use irradiation source of even light intensity at whole irradiation range, since size and shape of NPs influence wavelength of LSPR;

(iii) determine an optimal amount of noble metal deposited on support.

The last point (iii) seems the most difficult for realization since two opposite relationships for small and large gold NPs were observed, that is, parabolic dependence for small gold NPs and cascade dependence for large gold NPs. In this regard, small amount of gold ( $\ll 2 \mathrm{wt} \%)$ should be deposited on small anatase titania to obtain optimal light absorption. While for larger gold NPs deposited on large rutile titania there is no optimal amount of gold (cascade increase) and due to economic reasons the first plateau could be the most recommended for application, that is, $0.5 \mathrm{wt} \%$.

\section{Acknowledgments}

This research was financially supported by KAKENHI (Grant-in-Aid for Scientific Research) on Priority Area "Strong Photon-Molecule Coupling Fields" (no. 470), the Global Center of Excellence (GCOE) Program "Catalysis as the Basis for Innovation in Material Science," and Hokkaido Innovation through Nanotechnology Support (HINTS) of the Ministry of Education, Science, Culture and Sports of Japan. E. Kowalska acknowledges the European Commission for Marie Curie FP7-Reintegration Grant (IRG) FP7-248666 and Ministry of Science, Research and the Arts of BadenWürttemberg for Brigitte Schlieben-Lange-Programme.

\section{References}

[1] M. R. Hoffmann, S. T. Martin, W. Choi, and D. W. Bahnemann, "Environmental applications of semiconductor photocatalysis," Chemical Reviews, vol. 95, no. 1, pp. 69-96, 1995.

[2] A. Fujishima, K. Hashimoto, and T. Watanabe, $\mathrm{TiO}_{2}$ Photocatalysis. Fundamentals and Applications, BKC Inc., Tokyo, Japan, 1999.

[3] H. Kisch, Heterogeneous Catalysis in Organic Chemistry, edited by G. V. Smith and F. Notheisz, 2000.
[4] T. Ohno, M. Akiyoshi, T. Umebayashi, K. Asai, T. Mitsui, and M. Matsumura, "Preparation of S-doped $\mathrm{TiO}_{2}$ photocatalysts and their photocatalytic activities under visible light," Applied Catalysis A, vol. 265, no. 1, pp. 115-121, 2004.

[5] D. Mitoraj and H. Kisch, "On the mechanism of urea-induced titania modification,” Chemistry, vol. 16, no. 1, pp. 261-269, 2010.

[6] P. Zabek and H. Kisch, "Polyol-derived carbon-modified titania for visible light photocatalysis," Journal of Coordination Chemistry, vol. 63, no. 14-16, pp. 2715-2726, 2010.

[7] A. Zaleska, P. Górska, J. W. Sobczak, and J. Hupka, "Thioacetamide and thiourea impact on visible light activity of $\mathrm{TiO}_{2}$," Applied Catalysis B, vol. 76, no. 1-2, pp. 1-8, 2007.

[8] R. Asahi, T. Morikawa, T. Ohwaki, K. Aoki, and Y. Taga, "Visible-light photocatalysis in nitrogen-doped titanium oxides," Science, vol. 293, no. 5528, pp. 269-271, 2001.

[9] T. Ohno, F. Tanigawa, K. Fujihara, S. Izumi, and M. Matsumura, "Photocatalytic oxidation of water on $\mathrm{TiO}_{2}$-coated $\mathrm{WO}_{3}$ particles by visible light using Iron(III) ions as electron acceptor," Journal of Photochemistry and Photobiology A, vol. 118, no. 1, pp. 41-44, 1998.

[10] H. Kisch, G. Burgeth, and W. Macyk, "Visible light photocatalysis by a titania transition metal complex," Advances in Inorganic Chemistry, vol. 56, pp. 241-259, 2004.

[11] B. Kraeutler and A. J. Bard, "Heterogeneous photocatalytic preparation of supported catalysts. Photodeposition of platinum on $\mathrm{TiO}_{2}$ powder and other substrates," Journal of the American Chemical Society, vol. 100, no. 13, pp. 4317-4318, 1978.

[12] G. A. Hope and A. J. Bard, "Platinum/titanium dioxide (rutile) interface. Formation of ohmic and rectifying junctions," Journal of Physical Chemistry, vol. 87, no. 11, pp. 1979-1984, 1983.

[13] P. Pichat, J. M. Herrmann, J. Disdier, H. Courbon, and M. N. Mozzanega, "Photocatalytic hydrogen production from aliphatic alcohols over a bifunctional platinum on titanium dioxide catalyst," Nouveau Journal de Chimie, vol. 5, no. 12, pp. 627-636, 1981.

[14] B. Ohtani, M. Kakimoto, S. Nishimoto, and T. Kagiya, "Photocatalytic reaction of neat alcohols by metal-loaded titanium(IV) oxide particles," Journal of Photochemistry and Photobiology, A, vol. 70, no. 3, pp. 265-272, 1993.

[15] E. Kowalska, H. Remita, C. Colbeau-Justin, J. Hupka, and J. Belloni, "Modification of titanium dioxide with platinum ions and clusters: application in photocatalysis," Journal of Physical Chemistry C, vol. 112, no. 4, pp. 1124-1131, 2008.

[16] T. Sakata and T. Kawai, "Heterogeneous photocatalytic production of hydrogen and methane from ethanol and water," Chemical Physics Letters, vol. 80, no. 2, pp. 341-344, 1981.

[17] Y. Tian and T. Tatsuma, "Mechanisms and applications of plasmon-induced charge separation at $\mathrm{TiO}_{2}$ films loaded with gold nanoparticles," Journal of the American Chemical Society, vol. 127, no. 20, pp. 7632-7637, 2005.

[18] E. Kowalska, R. Abe, and B. Ohtani, "Visible light-induced photocatalytic reaction of gold-modified titanium(IV) oxide particles: action spectrum analysis," Chemical Communications, no. 2, pp. 241-243, 2009.

[19] H. Kominami, A. Tanaka, and K. Hashimoto, "Gold nanoparticles supported on cerium(IV) oxide powder for mineralization of organic acids in aqueous suspensions under irradiation of visible light of $\lambda=530 \mathrm{~nm}$," Applied Catalysis A, vol. 397, no. 1-2, pp. 121-126, 2011.

[20] A. Zielińska, E. Kowalska, J. W. Sobczak et al., "Silver-doped $\mathrm{TiO}_{2}$ prepared by microemulsion method: surface properties, 
bio- and photoactivity," Separation and Purification Technology, vol. 72, no. 3, pp. 309-318, 2010.

[21] M. Haruta, "Size- and support-dependency in the catalysis of gold," Catalysis Today, vol. 36, no. 1, pp. 153-166, 1997.

[22] R. Zanella, S. Giorgio, C. H. Shin, C. R. Henry, and C. Louis, "Characterization and reactivity in $\mathrm{CO}$ oxidation of gold nanoparticles supported on $\mathrm{TiO}_{2}$ prepared by depositionprecipitation with $\mathrm{NaOH}$ and urea," Journal of Catalysis, vol. 222, no. 2, pp. 357-367, 2004.

[23] A. Furube, L. Du, K. Hara, R. Katoh, and M. Tachiya, "Ultrafast plasmon-induced electron transfer from gold nanodots into $\mathrm{TiO}_{2}$ nanoparticles," Journal of the American Chemical Society, vol. 129, no. 48, pp. 14852-14853, 2007.

[24] L. Du, A. Furube, K. Hara, R. Katoh, and M. Tachiya, "Plasmon induced electron transfer at gold- $\mathrm{TiO}_{2}$ interface under femtosecond near-IR two-photon excitation," Thin Solid Films, vol. 518, no. 2, pp. 861-864, 2009.

[25] E. Kowalska, O. O. P. Mahaney, R. Abe, and B. Ohtani, "Visible-light-induced photocatalysis through surface plasmon excitation of gold on titania surfaces," Physical Chemistry Chemical Physics, vol. 12, no. 10, pp. 2344-2355, 2010.

[26] C. G. Silva, R. Juá rez, T. Marino, R. Molinari, and H. García, "Influence of excitation wavelength (UV or visible light) on the photocatalytic activity of titania containing gold nanoparticles for the generation of hydrogen or oxygen from water," Journal of the American Chemical Society, vol. 133, no. 3, pp. 595-602, 2011.

[27] D. B. Ingram and S. Linic, "Water splitting on composite plasmonic-metal/semiconductor photoelectrodes: evidence for selective plasmon-induced formation of charge carriers near the semiconductor surface," Journal of the American Chemical Society, vol. 133, no. 14, pp. 5202-5205, 2011.

[28] Y. Ide, N. Nakamura, H. Hattori et al., "Sunlight-induced efficient and selective photocatalytic benzene oxidation on $\mathrm{TiO}_{2}$-supported gold nanoparticles under $\mathrm{CO}_{2}$ atmosphere," Chemical Communications, vol. 47, pp. 11531-11533, 2011.

[29] S. C. Warren and E. Thimsen, "Plasmonic solar water splitting," Energy \& Environmental Science, vol. 5, pp. 51335146, 2012.

[30] V. Mizeikis, E. Kowalska, and S. Juodkazis, "Resonant, localization, enhancement, and polarization o optical fields in nanoscale interface regions for photo-catalytic application," Journal of Nanoscience and Nanotechnology, vol. 11, pp. 2814-2822, 2011.

[31] L. Rosa, S. Juodkazis, and E. Kowalska, "Localized photocatalysis by Au-titania plasmonics," in Proceedings of the International Workshop on Biophotonics (BioPhotonics '11), pp. 1-3, Parma, Italy, 2011.

[32] A. Sanchez, S. Abbet, U. Heiz et al., "When gold is not noble: nanoscale gold catalysts," Journal of Physical Chemistry A, vol. 103, no. 48, pp. 9573-9578, 1999.

[33] M. M. Schubert, S. Hackenberg, A. C. Van Veen, M. Muhler, V. Plzak, and J. J. Behm, "CO oxidation over supported gold catalysts -"Inert" and "active" support materials and their role for the oxygen supply during reaction," Journal of Catalysis, vol. 197, no. 1, pp. 113-122, 2001.

[34] A. Zielińska-Jurek, E. Kowalska, J. W. Sobczak, W. Lisowski, B. Ohtani, and A. Zaleska, "Preparation and characterization of monometallic $(\mathrm{Au})$ and bimetallic $(\mathrm{Ag} / \mathrm{Au})$ modified-titania photocatalysts activated by visible light," Applied Catalysis B, vol. 101, no. 3-4, pp. 504-514, 2011.

[35] E. Kowalska, L. Rosa, S. Rau, S. Juodkazis, and B. Ohtani, "Preparation of titania/Au/titania nanoparticles with improved photoactivity and stability for visible-light application," In press.

[36] E. Kowalska and S. Rau, "Photoreactors for wastewater treatment: a review," Recent Patents on Engineering, vol. 4, no. 3, pp. 242-266, 2010.

[37] O. O. Prieto-Mahaney, N. Murakami, R. Abe, and B. Ohtani, "Correlation between photoeatalytic activities and structural and physical properties of titanium(IV) oxide powders," Chemistry Letters, vol. 38, no. 3, pp. 238-239, 2009.

[38] K. Esumi, S. Sarashina, and T. Yoshimura, "Synthesis of gold nanoparticles from an organometallic compound in supercritical carbon dioxide," Langmuir, vol. 20, no. 13, pp. 5189-5191, 2004.

[39] H. Yuzawa, T. Yoshida, and H. Yoshida, "Gold nanoparticles on titanium oxide effective for photocatalytic hydrogen formation under visible light," Applied Catalysis B, vol. 115-116, pp. 294-302, 2012.

[40] P. Johne and H. Kisch, "Photoreduction of carbon dioxide catalysed by free and supported zinc and cadmium sulphide powders," Journal of Photochemistry and Photobiology A, vol. 111, no. 1-3, pp. 223-228, 1997.

[41] J. M. Herrmann, "Heterogeneous photocatalysis: fundamentals and applications to the removal of various types of aqueous pollutants," Catalysis Today, vol. 53, no. 1, pp. 115129, 1999.

[42] D. Curcó, J. Giménez, A. Addardak, S. Cervera-March, and S. Esplugas, "Effects of radiation absorption and catalyst concentration on the photocatalytic degradation of pollutants," Catalysis Today, vol. 76, no. 2-4, pp. 177-188, 2002.

[43] H. Kisch, "On the problem of comparing rates or apparent quantum yields in heterogeneous photocatalysis," Angewandte Chemie, vol. 49, no. 50, pp. 9588-9589, 2010.

[44] B. Ohtani, M. Kakimoto, H. Miyadzu, S. I. Nishimoto, and T. Kagiya, "Effect of surface-adsorbed 2-propanol on the photocatalytic reduction of silver and/or nitrate ions in acidic $\mathrm{TiO}_{2}$ suspension," Journal of Physical Chemistry, vol. 92, no. 20, pp. 5773-5777, 1988.

[45] N. Dimitratos, A. Villa, C. L. Bianchi, L. Prati, and M. Makkee, "Gold on titania: effect of preparation method in the liquid phase oxidation," Applied Catalysis A, vol. 311, no. 1-2, pp. 185-192, 2006.

[46] S. Sakthivel, M. V. Shankar, M. Palanichamy, B. Arabindoo, D. W. Bahnemann, and V. Murugesan, "Enhancement of photocatalytic activity by metal deposition: characterisation and photonic efficiency of Pt, $\mathrm{Au}$ and Pd deposited on $\mathrm{TiO}_{2}$ catalyst," Water Research, vol. 38, no. 13, pp. 3001-3008, 2004.

[47] H. Li, Z. Bian, J. Zhu, Y. Huo, H. Li, and Y. Lu, "Mesoporous $\mathrm{Au} / \mathrm{TiO}_{2}$ nanocomposites with enhanced photocatalytic activity," Journal of the American Chemical Society, vol. 129, no. 15, pp. 4538-4539, 2007.

[48] A. Orlov, D. A. Jefferson, N. Macleod, and R. M. Lambert, "Photocatalytic properties of $\mathrm{TiO}_{2}$ modified with gold nanoparticles in the degradation of 4-chlorophenol in aqueous solution," Catalysis Letters, vol. 92, no. 1-2, pp. 41-47, 2004.

[49] B. Tian, J. Zhang, T. Tong, and F. Chen, "Preparation of $\mathrm{Au} / \mathrm{TiO}_{2}$ catalysts from $\mathrm{Au}(\mathrm{I})$-thiosulfate complex and study of their photocatalytic activity for the degradation of methyl orange," Applied Catalysis B, vol. 79, no. 4, pp. 394-401, 2008.

[50] M. V. Dozzi, L. Prati, P. Canton, and E. Selli, "Effects of gold nanoparticles deposition on the photocatalytic activity of titanium dioxide under visible light," Physical Chemistry Chemical Physics, vol. 11, no. 33, pp. 7171-7180, 2009. 

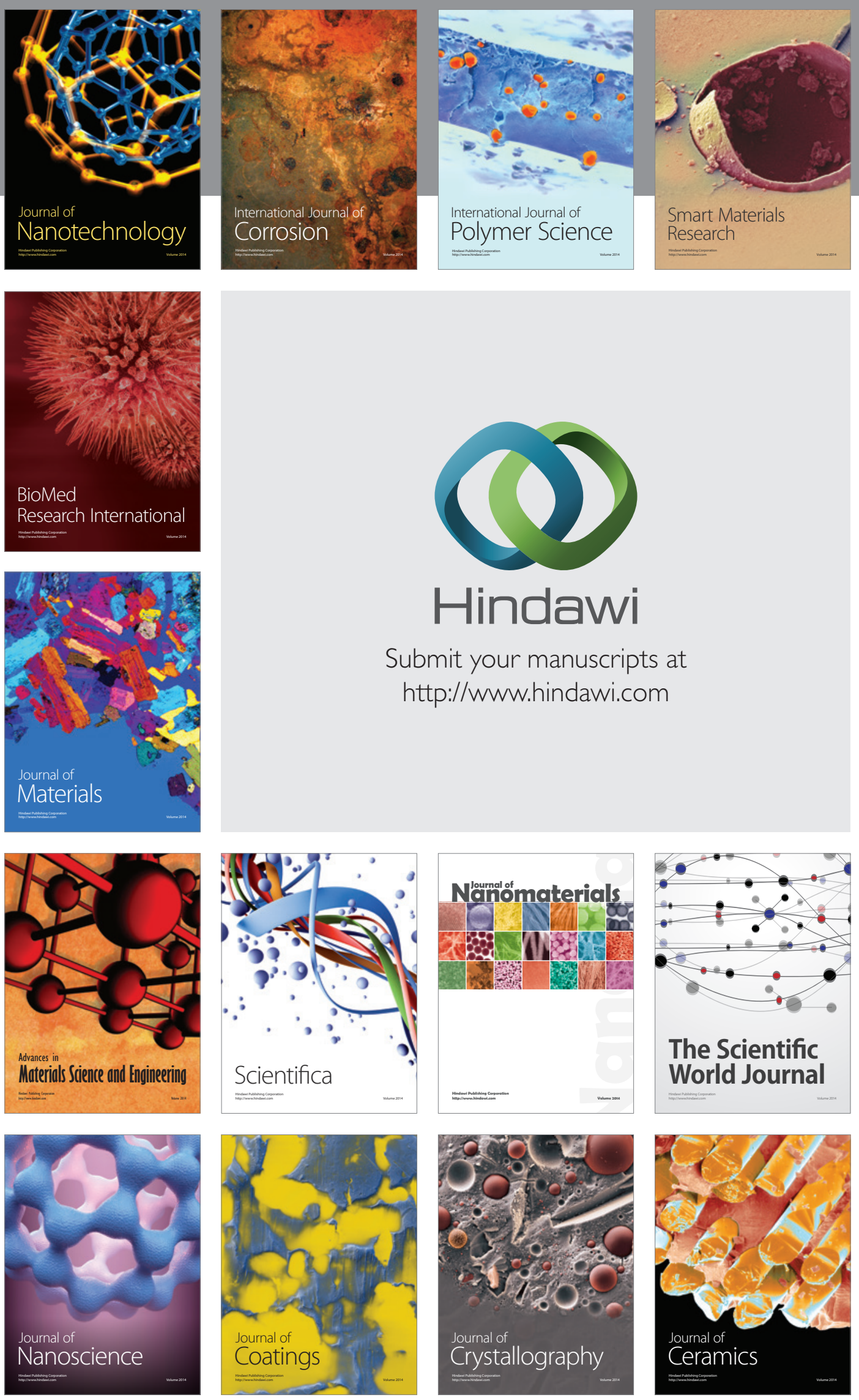

The Scientific World Journal

Submit your manuscripts at

http://www.hindawi.com

\section{World Journal}

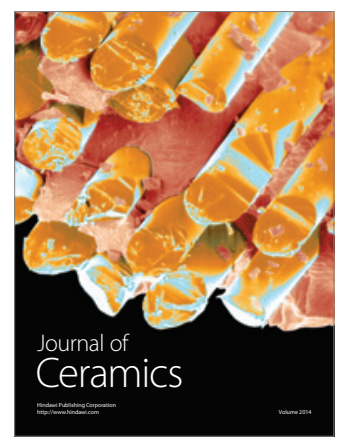

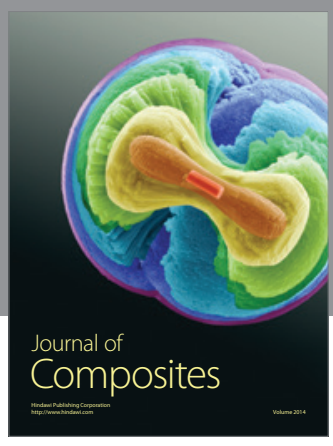
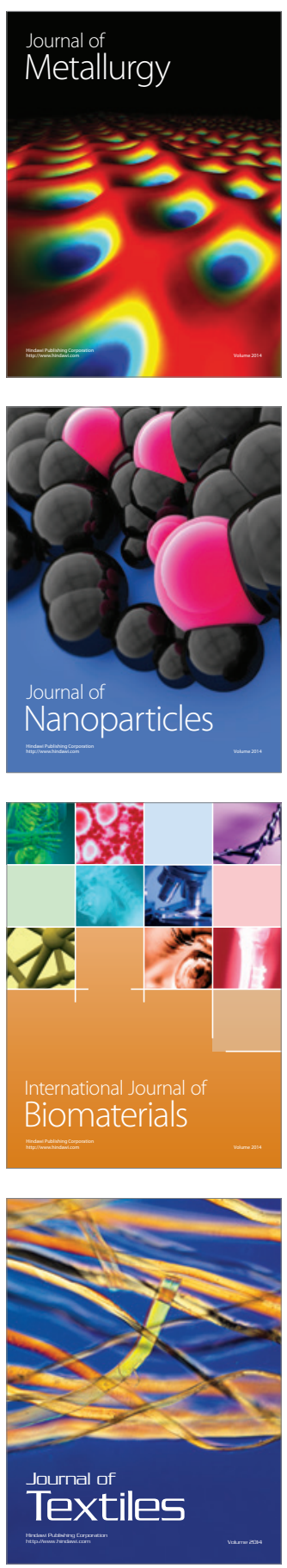